\title{
Case Report and Review of the Literature
}

\section{Decreased Heart Rate Variability in COVID-19}

\section{Chengfen Yin ${ }^{1,2}$, Jianguo $\mathrm{Li}^{3}$, Zhiyong Wang ${ }^{2}$, Yongle $\mathrm{Zhi}^{2}$ and Lei $\mathrm{Xu}^{2 *}$}

${ }^{1}$ The Third Central Clinical College of Tianjin Medical University, Tianjin, China

${ }^{2}$ Department of Critical Care Medicine, Tianjin Third Central Hospital, Tianjin Key Laboratory of Extracorporeal Life Support for Critical Diseases, Artificial Cell Engineering Technology Research Center, Tianjin Institute of Hepatobiliary Disease, Tianjin, China

${ }^{3}$ Department of Respiratory and Critical Medicine, Tianjin Haihe Hospital, Tianjin, China

\section{A R T ICLE INFO \\ Article history: \\ Received: 7 October, 2021 \\ Accepted: 23 October, 2021 \\ Published: 13 December, 2021 \\ Keywords: \\ The coronavirus disease 2019 \\ angiotensin-converting enzyme 2 \\ heart rate variability \\ severe acute respiratory syndrome \\ coronavirus 2}

\begin{abstract}
A B S T R AC T
On March 12, 2020, the World Health Organization (WHO) announced that the coronavirus disease 2019 (COVID-19) outbreak had become a pandemic. COVID-19 is caused by severe acute respiratory syndrome coronavirus 2 (SARS-CoV-2), which primarily infects the lower airways and binds to AngiotensinConverting Enzyme 2 (ACE2) on alveolar epithelial cells. ACE2 is widely expressed, not only in the lungs but also in the cardiovascular system. Therefore, SARS-CoV-2 can also damage the myocardium. We analysed three COVID-19 cases that resulted in death and found that either COVID-19 or antiviral drugs could affect the coupling between the autonomic nervous system and the sinus node, thus affecting heart rate variability and preventing the heart rate from rising in response to the increase in body temperature. Early detection of the preclinical phase of cardiac autonomic dysfunction may help determine patients in need of aggressive treatment and control of cardiovascular risk factors. Antiviral drugs should be used with caution in patients with heart injury.
\end{abstract}

1.

\footnotetext{
${ }^{*}$ Correspondence to: Lei Xu, Department of Critical Care Medicine, Tianjin Third Central Hospital, Artificial Cells Key Laboratory of Tianjin, Tianjin Institute of Hepatobiliary Disease, Artificial Cell Engineering Technology Research Center of Public Health Ministry, Tianjin 300170, China; E-mail: nokia007008@163.com 